\title{
ACTIVIDADES DE PROGRAMACIÓN NEUROLINGÜÍSTICA COMO FUENTE DE AFRONTAMIENTO DEL ESTRÉS LABORAL EN PERSONAL DOCENTE DE LA UNIDAD EDUCATIVA PRINCETON
}

\author{
NEUROLINGUISTIC PROGRAMMING ACTIVITIES AS A SOURCE OF FACULTY \\ OF LABOR STRESS IN TEACHING STAFF OF PRINCETON EDUCATIONAL UNIT
}

ALEJANDRO HUILCA ÁLVAREZ

Escuela Superior Politécnica de Chimborazo, Ecuador. alnickwil@gmail.com

RESUMEN

Con la finalidad de constatar un cambio en la percepción de las personas respecto a la intensidad del estrés laboral, el presente artículo recoge los datos de evaluación de la intervención realizada en el personal docente de la Unidad Educativa Princeton de la ciudad de Riobamba, Ecuador. Los procedimientos de evaluación se realizaron mediante la aplicación de encuestas de estrés laboral validadas. Dicha evaluación se efectuó antes y después de la aplicación de la Programación Neurolingüística con el objetivo de verificar un cambio de percepción de los docentes hacia las preguntas de cada encuesta. El estudio se aplicó a los 17 docentes de la institución. Las sesiones de Programación Neurolingüística se realizaron cada viernes durante 12 semanas. Los resultados expresaron cambios significativos en la encuesta denominada Cuestionario de Problemas Psicosomáticos que mide la percepción de factores de salud. Por otra parte, se produjo un cambio muy leve en la percepción de la encuesta denominada Escala General de Satisfacción que mide la percepción de los factores laborales. Se aplicó el método estadístico chi - cuadrado al resultado global de cada encuesta, resultando la aceptación de la hipótesis que responde afirmativamente al afrontamiento del estrés laboral en la unidad educativa.

PALABRAS CLAVE: programación neurolingüística, percepción, estrés laboral, unidades educativas.

\begin{abstract}
With the objective of verifying a change in the perception of the people regarding the intensity of work stress, this article collects the evaluation data of the intervention performed in the teaching staff of the Princeton Educational Unit of the city of Riobamba, Ecuador. The evaluation procedures were performed through the application of validated work stress surveys. This evaluation was done before and after the application of the Neurolinguistic Programming with the objective of verifying a change of perception of the teachers to the questions of each survey. The study was applied to the 17 teachers of the institution. The Neurolinguistic Programming sessions were held every Friday for 12 weeks. The results expressed significant changes in the survey called Psychosomatic Problems Questionnaire that measures the perception of health factors. On the other hand, there was a very slight change in the perception of the survey called the General Satisfaction Scale that measures the perception of labor factors. The chi - square statistical method was applied to the overall result of each survey, resulting in the acceptance of the hypothesis that responds affirmatively to coping with work stress in the educational unit.
\end{abstract}

KEYWORDS: neuro-linguistic Programming, perception, work stress, schools. 


\section{INTRODUCCIÓN}

Una de las finalidades de los directivos de las instituciones educativas de nivel primario y secundario es velar por el bienestar integral de las personas que conforman la comunidad educativa. La gestión educativa se considera como un proceso continuo que busca alcanzar el bienestar colectivo de una comunidad estudiantil (Valenzuela, Ramírez y Alfaro, 2016). Uno de los problemas que más preocupan al profesorado de instituciones educativas son las deficiencias en el estado de su salud derivadas directamente del tipo de funciones que desempeñan (Gairín, Moles, Castro, Alegre, Sans, Rosales y Lorenzo, 2012). Entre los factores más comunes, causantes de la afectación de la salud del profesorado secundario y primario es el estrés. Este último, se considera una reacción fisiológica del organismo humano en el que los mecanismos de defensa de las personas entran en juego ante situaciones agobiantes o amenazantes (Peiró, 2011). Las consecuencias del estrés laboral no solo se limitan a un círculo profesional sino que también afectan las acciones personales, tanto familiares como sociales (Sánchez, 2011).

Las instituciones educativas, al igual que todas las organizaciones laborales se encuentran en permanente evolución, pero ante ello se generan nuevas metas educativas que deben ser alcanzadas. En la actualidad los docentes están sometidos a una serie de cambios impulsados por las reformas educativas (Velázquez, García, Díaz, Rodríguez y Ávila, 2014).

La labor en las instituciones educativas es considerada demandante como en muchas otras ramas laborales. La importancia creciente del estrés laboral está relacionada con las transformaciones que se están produciendo en los mercados de trabajo.

Bajo este contexto, las instituciones educativas empiezan a mirar estrategias para afrontar los factores estresantes. Estas, suelen ser de dos tipos: centradas en el problema (cambiar la situación y resolver el problema) y las centradas en la emoción (controlar el estrés emocional que genera la situación) (Acosta y Burgillos, 2014).

Las estrategias de tipo emocional se centran en aplicar metodologías cognitivas como por ejemplo la psicología positiva, que es un buen método para reducir el estrés (Peralta, Besio, Rubio, Atabales y Salinas, 2016). Este modelo puede enfocarse a los docentes que cumplen exigencias familiares (cuidado de familiares, actividades domésticas) y del establecimiento en el cual laboran.
Por otro lado las instituciones educativas han implementado estrategias en el proceso y modelo de enseñanza como implementación de las TIC (tecnologías de la información y comunicación) esta forma de trabajo permite realizar actividades colaborativas - cooperativas, disminuyendo el trabajo manual y monótono, mitigando el estrés. (Antolín y Santoro, 2016, p.7).

Una de las metodologías empleadas para mejorar los procesos emocionales en las personas es la Programación Neurolingüística. Esta estrategia es usada como una aplicación práctica que permite desarrollar habilidades para el crecimiento personal, la mejora de las relaciones interpersonales, y para conocer la percepción de nosotros mismos y de los demás (Fuentetaja, 2014).

"La Programación Neurolingüística parte de los fundamentos del constructivismo, en los que se define la realidad como una invención y no como un descubrimiento" (Peñafiel y Kenia, 2017, p.19). Es decir, esta estrategia se basa en que las personas observan la realidad como una percepción. Esta última, vista de otra manera, generaría pensamientos y emociones diferentes si se aplicasen las estrategias emocionales adecuadas.

La Programación Neurolingüística utiliza un sin número de técnicas para lograr modificar los pensamientos negativos de las personas y otórgales otro enfoque, entre ellos por ejemplo la técnica del anclaje, que permite acceder de manera rápida y segura a los puntos clave de las personas (Colorado, 2013, p.30).

Los conferencistas que realizan sesiones de motivación la utilizan fundamentalmente para contrarrestar el estrés laboral. Muchos autores ubican varias técnicas como parte de las estrategias de la Programación Neurolinguística, entre las principales destacan el equilibrio de los sistemas representacionales; la modificación de creencias; análisis de metaprogramas; y la comunicación efectiva:

Equilibrio de los sistemas representacionales. Los sistemas representacionales clasifican a las personas mediante su forma de aprendizaje, catalogándolos en: auditivo, visual y kinestésico (Agüera, Medina, Diz, Camacho, Tovar y Escribano, 2016). La importancia de su equilibrio radica en mejorar la manera de percibir las situaciones, cambiando la percepción de los sistemas deficientes, sean estos auditivos, visuales o kinestésicos.

Modificación de creencias. Las creencias son consideradas como ideas fuertemente 
arraigadas y verdaderas a las que las personas se aferran sin poner en tela de juicio (Lizarraga, 2014). La importancia de su modificación radica en moldear la mente de tal manera que la percepción respecto a situaciones de la vida cotidiana, sea más real y optimista.

Buen manejo de los metaprogramas. Los metaprogramas se consideran como filtros que procesan la realidad. "También se pueden entender como tendencias de percepción y organización de los datos de la realidad" (Dobrinsky, 2012, p. 40). La importancia de su buen manejo es fundamental a la hora percibir eficientemente las semejanzas, ausencias, disociaciones y procedimientos que la mente realiza automáticamente.

La comunicación efectiva. Puede contribuir a construir relaciones más fuertes, y profundas. La comunicación es esencial a la hora de vincular una idea con objetivos claros en un grupo en particular (Bavister y Vickers, 2005).

PLAN DE ACCIÓN

El plan de acción desarrollado, consistió en ejecutar sesiones grupales de aplicación de estrategias de Programación Neurolingüística a los y las docentes de la Unidad Educativa Princeton. Tuvo como finalidad analizar el comportamiento del estrés laboral y su influencia en el actuar diario de los y las docentes en el ambiente de trabajo. Para ello, también se realizó una medición del estrés laboral mediante encuestas para verificar si existió una variación en las mismas. Esta evaluación se efectuó antes del inicio del programa de sesiones grupales y al culminar el estudio.

Dichas estrategias se basaron en 4 aspectos fundamentales de la programación neurolingüística: equilibrio del sistema representacional, modificación de creencias, manejo de los metaprogramas y comunicación efectiva.

MÉTODOS

El estudio fue desarrollado en toda la población docente de la Unidad Educativa Princeton, considerando que la cantidad de docentes no es un número sustancioso para tomar una muestra (17 personas).

La técnica de medición del estrés laboral en el personal docente de la Unidad Educativa Princeton, se ejecutó mediante la aplicación de dos encuestas validadas de estrés laboral: I) el Cuestionario de Problemas Psicosomáticos, adaptación española del trabajo de Hock (1988), realizada por García, Castellón, Alba- dalejo y García (1993); y II) la Escala General de Satisfacción, adaptación a la obra Overall Job Satisfaction de Warr, (Cook y Wall, 1979), realizada por Pérez y Fidalgo (1995).

Cabe recalcar que el primer cuestionario mencionado evalúa la insatisfacción de las personas respecto a la percepción de su nivel de salud; y el segundo evalúa la insatisfacción de las personas respecto a la percepción de las condiciones intrínsecas de su trabajo.

Usualmente estas encuestas son utilizadas juntas como dos partes de una misma encuesta. Su tabulación se realizó mediante la clasificación del nivel de satisfacción.

Las encuestas se aplicaron a los 17 docentes de la institución, una vez al inicio y otra vez al final del estudio con el orden de las preguntas cambiado. Luego se procedió a aplicar el análisis comparativo de los resultados tabulados.

Las Estrategias de Programación Neurolingüística, así como las encuestas, fueron aplicadas bajo un cronograma específico y por una profesional en psicología, con la ayuda de un profesional en seguridad industrial y prevención de riesgos laborales, durante 12 semanas consecutivas.

PROCEDIMIENTO

A continuación, en la Tabla 1 se muestra el cronograma de sesiones y actividades grupales de Programación Neurolingüística que se realizó.

\section{TABLA 1. CRONOGRAMA DE ACTIVIDADES GRUPALES}

\begin{tabular}{|c|c|c|c|}
\hline NO. & SEMANA & FECHA & ACTIVIDAD \\
\hline 1 & Semana 1 & $11 / 01 / 2017$ & $\begin{array}{l}\text { Aplicación de las encuestas de estrés } \\
\text { y explicación general de los temas y } \\
\text { sesiones que se realizarán. }\end{array}$ \\
\hline 2 & Semana 2 & 19/01/2017 & $\begin{array}{l}\text { Identificación de los sistemas represen- } \\
\text { tacionales (visual, auditivo, quinestésico). } \\
\text { Ejercicios para el equilibrio de los mismos. }\end{array}$ \\
\hline 3 & Semana 3 & 27/01/2017 & $\begin{array}{l}\text { Metaprogramas como filtros de la realidad } \\
\text { (parte 1). }\end{array}$ \\
\hline 4 & Semana 4 & $03 / 02 / 2017$ & $\begin{array}{l}\text { Metaprogramas como filtros de la realidad } \\
\text { (parte 2). }\end{array}$ \\
\hline 5 & Semana 5 & $08 / 02 / 2017$ & Estructura del pensamiento. \\
\hline 6 & Semana 6 & $16 / 02 / 2017$ & $\begin{array}{l}\text { Cambio de creencias - estructura del } \\
\text { pensamiento (parte 1). }\end{array}$ \\
\hline 7 & Semana 7 & 23/02/2017 & $\begin{array}{l}\text { Cambio de creencias - estructura del } \\
\text { pensamiento (parte 2). }\end{array}$ \\
\hline 8 & Semana 8 & 03/03/2017 & $\begin{array}{l}\text { Cambio de creencias y distorsiones (parte } \\
\text { 1). }\end{array}$ \\
\hline 9 & Semana 9 & $10 / 03 / 2017$ & $\begin{array}{l}\text { Cambio de creencias y distorsiones (parte } \\
\text { 2). }\end{array}$ \\
\hline 10 & Semana 10 & $15 / 03 / 2017$ & Estrategias y comunicación para el éxito. \\
\hline 11 & Semana 11 & $30 / 03 / 2017$ & Estrategias de comunicación. \\
\hline 12 & Semana 12 & $06 / 04 / 2017$ & $\begin{array}{l}\text { Evaluación de final de estrés, y conversa- } \\
\text { torio final de dudas y resultados. }\end{array}$ \\
\hline
\end{tabular}


Se procuró que dichas actividades no repercutieran en el desempeño docente ni en su horario de trabajo. Del mismo modo, se proporcionó el tiempo suficiente para culminar el programa planificado. Las actividades se programaron para ejecutarse un día por semana.

Como ya se ha mencionado, se aplicaron las encuestas de factores de salud (Cuestionario de problemas psicosomáticos) y condiciones laborales (Encuesta Escala General de Satisfacción); en este sentido, se alcanzó un solo resultado de las posibles opciones, lo cual se muestra a continuación en la Tabla 2.

A continuación, se explican detalladamente los posibles cambios en la percepción de las personas, tras culminar la aplicación del Programa de Programación Neurolingüística. Cabe recalcar que en los resultados del presente artículo se indica la opción de cambios de percepción resultante.

- Que, en la primera encuesta, se disminuya la percepción de insatisfacción respecto a las condiciones de salud y que se mantenga estable la percepción de insatisfacción de la segunda encuesta respecto a los factores laborales.

- Que en la primera encuesta se mantenga estable el resultado respecto a la insatisfacción de las condiciones de salud y que disminuya la percepción de la segunda encuesta respecto a la insatisfacción de las condiciones laborales.

- Que en la primera encuesta, se aumente la percepción de insatisfacción respecto a las condiciones de salud y que se mantenga estable la percepción de insatisfacción de la segunda encuesta respecto a los factores laborales.
- Que en la primera encuesta se mantenga estable el resultado respecto a la insatisfacción de las condiciones de salud y que aumente la percepción de la segunda encuesta respecto a la insatisfacción de las condiciones laborales.

- Que se mantengan estables los resultados en la aplicación de las 2 encuestas, antes y después de la ejecución del Programa de Programación Neurolingüística.

- Que los resultados de la primera encuesta, evidencien un incremento de la percepción respecto a las deficiencias de salud y que los resultados de la segunda encuesta muestren una disminución de la percepción respecto a la insatisfacción de factores laborales.

- Que los resultados de la primera encuesta indiquen una disminución de la percepción respecto a las deficiencias de salud y los resultados de la segunda encuesta muestren un aumento de la percepción respecto a la insatisfacción de los factores laborales.

- Que los resultados de ambas encuestas aumenten su percepción de insatisfacción, respecto a los factores de salud y condiciones de trabajo.

- Que los resultados de ambas encuestas disminuyan su percepción de insatisfacción respecto a los factores de salud y condiciones de trabajo.

Se considerará que un resultado se mantiene estable, si los resultados de las encuestas antes y después de la ejecución del Programa de Programación Neurolingüística no tuvieron variación.

tABLA 2. POSIBLES CAMBIOS EN LA PERCEPCIÓN DE DOCENTES RESPECTO A LAS ENCUESTAS APLICADAS, ANTES Y DESPUÉS DE LA INTERVENCIÓN DE PROGRAMACIÓN NEUROLINGUUÍSTICA

\begin{tabular}{|c|c|c|c|c|c|c|}
\hline \multirow[t]{2}{*}{\begin{tabular}{ll} 
& OPCIONES DE \\
$\stackrel{O}{9}$ & CAMBIOS DE \\
\multirow{J}{*}{} & PERCEPCIÓN
\end{tabular}} & \multicolumn{3}{|c|}{$\begin{array}{l}\text { FACTORES DE SALUD "CUESTIONARIO DE } \\
\text { PROBLEMAS PSICOSOMÁTICOS" } \\
\text { (POSIBLE CONCLUSIÓN DESPUÉS DE REALIZAR LA } \\
\text { COMPARACIÓN FINAL DEL ANTES Y DESPUÉS DE LA } \\
\text { INTERVENCIÓN) }\end{array}$} & \multicolumn{3}{|c|}{$\begin{array}{l}\text { CONDICIONES LABORALES. "ESCALA GENERAL DE } \\
\text { SATISFACCIÓN" } \\
\text { (POSIBLE CONCLUSIÓN DESPUÉS DE REALIZAR LA } \\
\text { COMPARACIÓN FINAL DEL ANTES Y DESPUÉS DE LA } \\
\text { INTERVENCIÓN) }\end{array}$} \\
\hline & $\begin{array}{l}\text { DISMINUYA LA } \\
\text { PERCEPCIÓN }\end{array}$ & $\begin{array}{l}\text { AUMENTE LA } \\
\text { PERCEPCIÓN }\end{array}$ & $\begin{array}{c}\text { SE MANTENGA ESTABLE } \\
\text { LA PERCEPCIÓN }\end{array}$ & \begin{tabular}{|l} 
DISMINUVA LA \\
PERCEPCIÓN
\end{tabular} & $\begin{array}{l}\text { AUMENTE LA } \\
\text { PERCEPCIÓN }\end{array}$ & $\begin{array}{c}\text { SE MANTENGA ESTABLE LA } \\
\text { PERCEPCIÓN }\end{array}$ \\
\hline A & $\mathrm{x}$ & & & & & $\mathrm{x}$ \\
\hline B & & & $\mathrm{x}$ & $\mathrm{x}$ & & \\
\hline C & & $\mathrm{x}$ & & & & $\mathrm{x}$ \\
\hline D & & & $x$ & & $\mathrm{x}$ & \\
\hline $\mathrm{E}$ & & & $\mathrm{x}$ & & & $\mathrm{x}$ \\
\hline $\mathrm{F}$ & & $\mathrm{x}$ & & $x$ & & \\
\hline$G$ & $\mathrm{x}$ & & & & $x$ & \\
\hline 1 & $\mathrm{x}$ & & & $\mathrm{x}$ & & \\
\hline
\end{tabular}


RESULTADOS

Tomando en cuenta la tabulación de lasw encuestas y analizando los posibles resultados expuestos anteriormente, podemos decir que la percepción de las personas respecto a ambas encuestas disminuyó. Cabe recalcar que en la primera encuesta (Cuestionario de Problemas Psicosomáticos), el cambio en la percepción fue considerable, mientras que, el cambio en la percepción de los docentes en la segunda encuesta (Escala General de Satisfacción) fue mínimo. Por consiguiente, tomando en cuenta las opciones mostradas en la Tabla 2, el resultado fue el literal "I".

La encuesta denominada Cuestionario de Problemas Psicosomáticos (encuesta 1) consta de 12 factores, relacionados a la salud, los cuales son mostrados en la Tabla 3.

\section{TABLA 3. ÍTEMS DEL CUESTIONARIO DE PROBLEMAS PSICOSOMÁTICOS}

\begin{tabular}{l|l|}
\hline $\begin{array}{l}\text { 1. Imposibilidad de conciliar el sueño. } \\
\text { 2. Jaquecas y dolores de cabeza. }\end{array}$ & $\begin{array}{l}\text { 8. Disminución del apetito. } \\
\text { sensación de ahogo. }\end{array}$ \\
\hline $\begin{array}{l}\text { 3. Indigestiones o molestias } \\
\text { gastrointestinales. }\end{array}$ & $\begin{array}{l}\text { 9. Temblores musculares (por } \\
\text { ejemplo tics nerviosos o parpadeos). }\end{array}$ \\
\hline $\begin{array}{l}\text { 4. Sensación de cansancio extremo o } \\
\text { agotamiento. }\end{array}$ & $\begin{array}{l}\text { 10. Pinchazos o sensaciones } \\
\text { dolorosas en distintas partes del } \\
\text { cuerpo. }\end{array}$ \\
\hline $\begin{array}{l}\text { 5. Tendencia de comer, beber o fumar } \\
\text { más de lo habitual. }\end{array}$ & $\begin{array}{l}\text { 11. Tentaciones fuertes de no } \\
\text { levantarse por la mañana. }\end{array}$ \\
\hline 6. Disminución del interés sexual. & $\begin{array}{l}\text { 12. Tendencias a sudar o } \\
\text { palpitaciones. }\end{array}$ \\
\hline
\end{tabular}

La encuesta muestra alternativas de respuesta como: nunca, casi nunca, pocas veces, algunas veces, con relativa frecuencia, con mucha frecuencia. Por ejemplo, al responder el primer Ítem de la Tabla 3, podemos observar que la respuesta más perturbadora, resulta si se elige la opción "con mucha frecuencia”, marcando así, la dirección de lo saludable y no saludable.

En la evaluación efectuada al finalizar el programa de sesiones grupales de Programación Neurolingüística, las personas encuestadas reaccionaron de diferentes formas al responder las preguntas, en comparación con la primera evaluación (al inicio del programa). El enfoque de respuestas cambió, disminuyendo las respuestas como: algunas veces, con relativa frecuencia, con mucha frecuencia.

A continuación, en la Tabla 4 se muestra el número de respuestas totales de cada alternativa antes y después de la aplicación del programa. Los resultados consolidados, muestran la tabulación total de cada ítem de respuesta que posee la encuesta (nunca, casi nunca, pocas veces, al- gunas veces, con relativa frecuencia, con mucha frecuencia).

TABLA 4. RESULTADOS CONSOLIDADOS DE LA ENCUESTA CUESTIONARIO DE PROBLEMAS PSICOSOMÁTICOS

\begin{tabular}{ccccccc}
\hline \multicolumn{2}{c}{ NUNCA (1) } & \multicolumn{2}{c}{ CASI NUNCA (2) } & \multicolumn{2}{c}{ POCAS VECES (3) } \\
\hline Antes & Después & Antes & Después & Antes & Después \\
\hline 43 & 57 & 74 & 116 & 111 & 123 \\
\hline \multicolumn{2}{|c}{ ALGUNAS VECES (4) } & \multicolumn{2}{c}{ CON RELATIVA } & \multicolumn{2}{c}{ CON MUCHA } \\
FRECUENCIA (5) & \multicolumn{2}{c}{ FRECUENCIA (6) } \\
\multicolumn{2}{|c|}{ Antes } & Después & Antes & Después & Antes & Después \\
180 & 124 & 137 & 74 & 60 & 12 \\
\hline
\end{tabular}

Como se observa existe una diferencia de resultados, si comparamos el antes y después, recalcando que las preguntas muestran la opción más optimista a la respuesta "Nunca" y la más pesimista a la opción "Con mucha frecuencia", entendiendo como una disminución de respuestas pesimistas y un aumento de respuestas optimistas. Gráficamente también se puede visualizar el porcentaje de cada opción como se muestra en las Figuras 1 y 2.

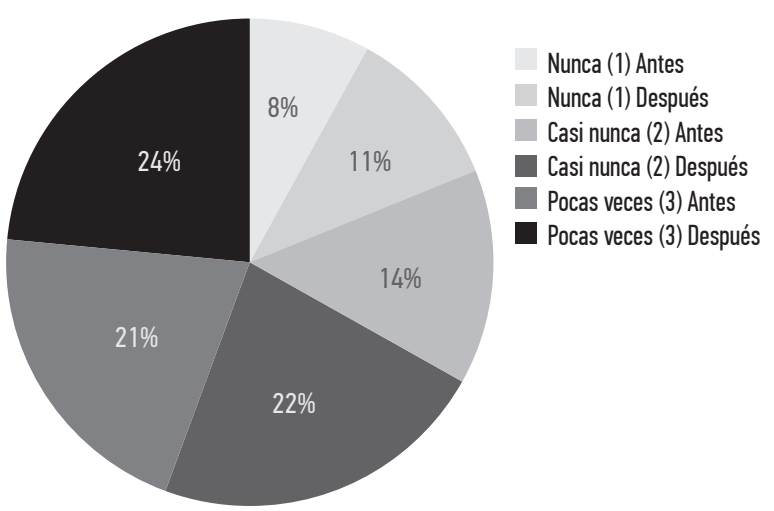

Figura 1. Resultados porcentuales del Cuestionario de Problemas Psicosomáticos, opciones de respuesta: Nunca, Casi nunca, Pocas veces.

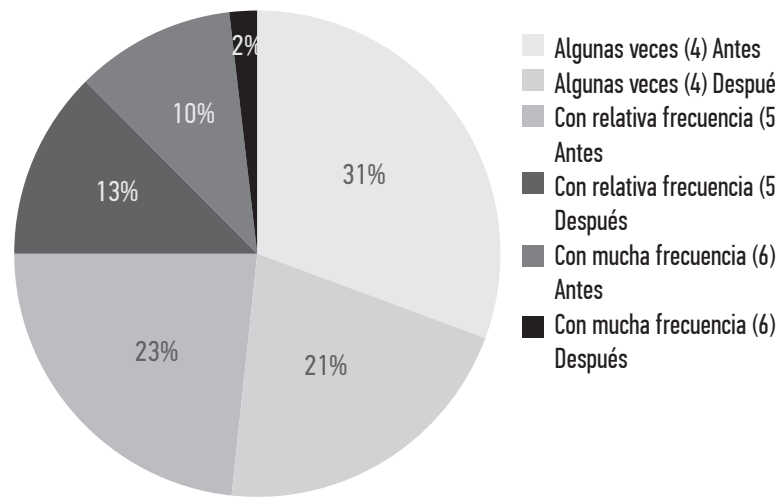

Figura 2. Resultados porcentuales del Cuestionario de Problemas Psicosomáticos, opciones de respuesta: Algunas veces, con relativa frecuencia, con mucha frecuencia

Por su parte, la encuesta denominada Escala General de Satisfacción (Encuesta 2) consta de 
15 factores, relacionados a los factores intrínsecos del trabajo según se muestra en la Tabla 5.

TABLA 5. ÍTEMS DE LA ENCUESTA ESCALA GENERAL DE SATISFACCIÓN (FACTORES DEL TRABAJO, ENCUESTA 2)

\begin{tabular}{l|l|}
$\begin{array}{l}\text { 1. Condiciones físicas de su trabajo. } \\
\text { 2. Libertad para elegir su propio } \\
\text { método de trabajo. }\end{array}$ & $\begin{array}{l}\text { 9. Relación entre dirección y } \\
\text { trabajadores en su empresa. }\end{array}$ \\
\hline $\begin{array}{l}\text { 3. Sus compañeros de trabajo. } \\
\text { 11. El modo en que su empresa está } \\
\text { gestionada. }\end{array}$ \\
\hline $\begin{array}{l}\text { 4. Reconocimiento que obtiene por el } \\
\text { trabajo bien hecho. }\end{array}$ & $\begin{array}{l}\text { 12. La atención que se presta a las } \\
\text { sugerencias que usted hace. }\end{array}$ \\
\hline $\begin{array}{l}\text { 5. Su superior inmediato. } \\
\text { 6. Responsabilidad que usted tiene } \\
\text { asignada. }\end{array}$ & $\begin{array}{l}\text { 14. Su horario de trabajo. } \\
\text { en su trabajo. }\end{array}$ \\
\hline $\begin{array}{l}\text { 7. Su salario. } \\
\text { 8. La posibilidad de utilizar sus } \\
\text { capacidades. }\end{array}$ & 15. Su estabilidad en el empleo. \\
\hline
\end{tabular}

El procedimiento del llenado de las encuestas muestra las opciones de respuesta por pregunta, con las siguientes alternativas a cada ítem: muy insatisfecho, insatisfecho, moderadamente insatisfecho, ni satisfecho ni insatisfecho, moderadamente satisfecho, satisfecho, muy satisfecho. Los resultados de la tabulación se muestran a continuación en la Tabla 6 .

La representación gráfica de los resultados es mostrada en la Figura 3.

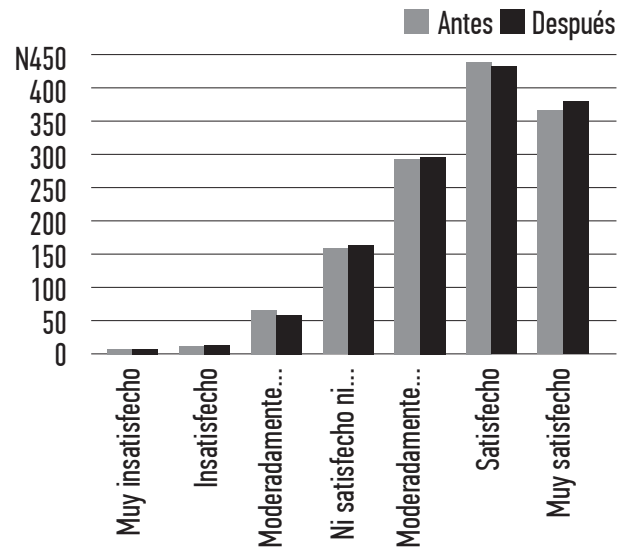

Figura 3. Resultados porcentuales de la encuesta "Escala General dVe Satisfacción"
Se pudo observar un pequeño cambio de resultados de cada ítem del cuestionario respecto a las alternativas de respuesta. La opción moderadamente satisfecho, aumentó 2 puntos, satisfecho disminuyó 6 puntos y muy satisfecho aumentó 14 puntos. Ello muestra un leve incremento de estas alternativas, después de la aplicación del programa de sesiones grupales de Programación Neurolingüística.

\section{COMPROBACIÓN ESTADÍSTICA}

Si nos planteamos una hipótesis referente a la efectividad de la Programación Neurolingüística, quedaría formulada de la siguiente forma: La aplicación de las sesiones grupales de estrategias de programación neurolingüística disminuyen la percepción de las personas respecto a la intensidad del estrés, mejorando su salud personal e incluso la percepción respecto a los factores laborales.

\section{RESULTADOS DEL PROCEDIMIENTO ESTADÍSTICO CHI - CUADRADO, APLICADO EN LAS ENCUESTAS DE ESTRÉS}

El resultado del chi cuadrado aplicado a la tabulación de respuestas, después de la aplicación del programa de sesiones grupales de Programación Neurolingüística, debe responder a la afirmación o negación de la efectividad del programa ejecutado.

El resultado del chi cuadrado del "Cuestionario de problemas psicosomáticos" es menor que su valor crítico, avalando la hipótesis respecto a la disminución de la percepción de factores perjudiciales de la salud provenientes del estrés según se muestra en la Tabla 7.

TABLA 7. RESULTADOS COMPARATIVOS DE LA APLICACIÓN DEL CHI - CUADRADO EN LA ENCUESTA "CONDICIONES DE SALUD" (ENCUESTA 1)

\begin{tabular}{|l|l|l|}
\hline$X^{2}$ calc & $\leq$ & valor crítico \\
\hline 1,357220 & $\leq$ & 11,0705 \\
\hline
\end{tabular}

El resultado del chi cuadrado del cuestionario "Escala general de satisfacción" es menor que su valor crítico avalando la hipótesis respecto a la disminución de la percepción a factores

TABLA 6. RESULTADOS DE LA TABULACIÓN GENERAL DE LA ENCUESTA "ESCALA GENERAL DE SATISFACCIÓN"

\begin{tabular}{|c|c|c|c|c|c|c|c|}
\hline \multicolumn{2}{|c|}{ MUY INSATISFECHO } & \multicolumn{2}{|c|}{ INSATISFECHO } & \multicolumn{2}{|c|}{ MODERADAMENTE INSATISFECHO } & \multicolumn{2}{|c|}{ MI SATISFECHO NI INSATISFECHO } \\
\hline ANTES & DESPUÉS & ANTES & DESPUÉS & ANTES & DESPUÉS & ANTES & DESPUÉS \\
\hline \multirow[t]{4}{*}{3} & 3 & 8 & 10 & 63 & 57 & 160 & 164 \\
\hline & MODERAD & ATISFECHO & & & MUY & ECHO & \\
\hline & ANTES & DESPUÉS & ANTES & DESPUÉS & ANTES & Después & \\
\hline & 297 & 299 & 444 & 438 & 371 & 385 & \\
\hline
\end{tabular}


laborales causantes del estrés según se muestra en la Tabla 8.

\section{TABLA 8. RESULTADOS COMPARATIVOS DE LA APLICACIÓN DEL CHI - CUADRADO EN LA ENCUESTA “ESCALA GENERAL DE SATISFACCIÓN" (ENCUESTA 2)}

\begin{tabular}{|l|l|l|}
\hline$X^{2}$ calc & $\leq$ & valor crítico \\
\hline 5,082983 & $\leq$ & 12,5916 \\
\hline
\end{tabular}

INTERPRETACIÓN DE RESULTADOS

Después de la aplicación de los cuestionarios se obtuvieron resultados diferenciados (antes - después de la aplicación del programa). En el Cuestionario de Problemas Psicosomáticos, el ítem "nunca” representa el nivel más favorable para los docentes y el ítem "con mucha frecuencia” representa el nivel más desfavorable. Se observó, que el consolidado de las cualificaciones cambió, disminuyendo las respuestas desfavorables para los docentes después de la aplicación del programa de sesiones de Programación Neurolingüística. En consecuencia, la percepción de las personas respecto a los factores que afectan negativamente su salud, mejoró en el transcurso de 12 semanas. Por ende, hubo un afrontamiento efectivo del estrés laboral.

Las alternativas en la encuesta denominada Escala General de Satisfacción, muestran el nivel más desfavorable con el resultado "muy insatisfecho" y el nivel más favorable con el resultado "muy satisfecho".

La variación de los resultados favorables fue mínima. A pesar de que en pocos ítems se incrementó el nivel de respuestas desfavorables, en la mayoría de ítems se incrementaron los resultados favorables, por lo que se puede precisar que la percepción positiva de los docentes hacia los factores laborales de la encuesta mejoró aunque muy levemente.

Tomando en cuenta los resultados de las 2 encuestas aplicadas, que son herramientas avaladas para la medición del estrés, concluimos que hubo una disminución de la percepción respecto a la intensidad del estrés en cada persona. Esto conlleva a asumir que las estrategias de Programación Neurolingüística fueron útiles a la hora de enfrentar el estrés en la Unidad Educativa Princeton de la ciudad de Riobamba, Ecuador.

Respecto al resultado estadístico utilizando el modelo Chi - cuadrado para el Cuestionario de Problemas Psicosomáticos, según el resultado se aceptaría la hipótesis. Es decir, sí hubo un cambio respecto a la percepción de los factores de salud medidos en la encuesta, afrontando efectivamente el estrés y recalcando lo dicho anteriormente.

También se observó el resultado estadístico de la encuesta "Escala General de Satisfacción", en donde se acepta la hipótesis respecto a un cambio positivo de los factores laborales, aunque recalcando que dicho cambio fue mínimo.

Conclusiones

La percepción de los docentes cambió positivamente respecto a su salud, esta conclusión se deriva de los resultados (antes - después) de la encuesta "Cuestionario de factores psicosomáticos", la cual está compuesta por factores relacionados al bienestar personal.

El cambio de percepción respecto a los factores laborales (evaluado en la encuesta Escala General de Satisfacción) no depende estrictamente del pensamiento subjetivo de las personas, ya que los aspectos que influyen en los factores del trabajo, involucran a terceras personas y condiciones impredecibles de la organización.

Se afrontó efectivamente el estrés laboral de los docentes de la Unidad Educativa Princeton, ya que las encuestas mostraron cambios (antes - después) en sus resultados.

\section{REFERENCIAS BIBLIOGRÁFICAS}

Acosta, M., y Burgillos, A. I. (2014). Estrés y burnout en profesores de primaria y secundaria de Huelva: las estrategias de afrontamiento como factor de protección. International Journal of Developmental andEducational Psychology, 4 (1), 303-310. Recuperado de http://dehesa.unex.es/bitstream/ handle/10662/1378/02149877_2014_1_4_303. pdf?sequence $=1$

Agüera, E., Medina, I., Diz-Pérez, J., Camacho, R., Tovar, P., y Escribano, B. (2016). Programación Neurolingüística: aprendizaje dinámico basado en el funcionamiento cerebral. Revista docencia veterinaria, 1, 18-29. Recuperado de http://www.vetdoc.es/index.php?journal=vet doc\&page $=$ article\&op=view\&path $\% 5 \mathrm{~B} \% 5 \mathrm{D}=9 \&$ path $\% 5 \mathrm{~B} \% 5 \mathrm{D}=2$

Antolín, B., y Santoro, C. (2016). Inteligencia emocional y TIC en Educación Infantil. Universidad de Sevilla. Granada España. Recuperado de https://idus.us.es/xmlui/handle/11441/43692

Bavister S., y Vickers, A. (2005). Programación Neurolingüística (PNL). Las claves para una comunicación más efectiva. Barcelona, España: Editorial Amat.

Colorado, C. (2013). Técnicas de programación neurolingüística como herramienta para la prevención y manejo del estrés laboral en la Secretaría Técnica del CIVE. Veracruz, México: Editorial de la Universidad Veracruzana. 
Dobrinsky, M. (2012). PNL La técnica del éxito. Buenos Aires: Ediciones Lea S.A.

Fuentetaja, A. (2014). Aportaciones de la Programación Neurolingüística a la educación. (Tesis de grado). Universidad de Valladolid, Valladolid, España. Recuperado de http://uvadoc.uva.es/handle/10324/5150

Gairín, J., Moles, R., Castro, D., Alegre, M., Sans, J., Rosales, M., y Lorenzo, M. (2012). La seguridad integral en los centros de enseñanza obligatoria en España. Seguridad y medio ambiente, 31 (121), 22-34. Recuperado de

http://www.belt.es/expertos/imagenes/seguridad $\% 20$ centros $\% 20$ ense $\%$ C3\%B1anza\%20obligatoria.pdf

García, M., Castellón, M., Albadalejo, M., y García, A. (1993). Relaciones entre Burnout, ambigüedad de rol y satisfacción laboral en el personal de Banca. Revista de Psicología del Trabajo y de las Organizaciones, 9(24), 17-26.

García, J. (2014). Cómo cambiar creencias con la PNL. Tesis Psicológica. Fundación Universitaria los Libertadores, 4 (1), 50 -51.

Lizarraga B. (2014) Identificación y modificación decreencias intermedias. Terapia cognitiva. Recuperado de http://sesionesdelaterapiacognitiva.blogspot. com/2014/03/identificacion-y-modificacionde.html

Peiró, J.M. (2011). Estrés laboral y riesgos psicosociales. Valencia, España: Universidad de Valencia.

Peñafiel, J., y Kenia, M. (2017). Estrategia de evaluación basada en la programación neurolingüística para la cátedra de Histología II de la carrera de Medicina de la UNIANDES. (Tesis de grado), Universidad Regional Autónoma de los Andes, Ambato, Ecuador. Recuperado de http://dspace.uniandes.edu.ec/ handle/123456789/5453
Peralta, A., Besio, C., Rubio, Y., Atabales, L., y Salinas, J. (2016). Efecto de un programa de psicología positiva e inteligencia emocional sobre la satisfacción laboral y vital. Revista Salud \& Sociedad, 1(2), 101-112. Recuperado de http://146.83.115.167/index.php/ saludysociedad/article/view/748/630

Pérez, J., y Fidalgo, M. (1993). Ministerio del trabajo y asuntos sociales. Instituto nacional de seguridad e Igiene en el Trabajo. NTP394 Escala General de Satisfacción. Madrid, España. Recuperado de

http://www.insht.es/InshtWeb/Contenidos/ Documentacion/FichasTecnicas/NTP/ Ficheros/301a400/ntp_394.pdf

Sánchez, F. (2011). Estrés laboral, satisfacción en el trabajo y bienestar psicológico en trabajadores de una industria cerealera. (Tesis de Grado). Universidad Abierta Interamericana, San Lorenzo, Argentina. Recuperado de http://imgbiblio.vaneduc. edu.ar/fulltext/files/TC111836.pdf

Valenzuela, J., Ramírez, M., y Alfaro, J. (2016). Construcción de indicadores institucionales para la mejora de la gestión y la calidad educativa. Revista Iberoamericana de Evaluación Educativa, 2 (2), 59-81. Recuperado de https://repositorio. uam.es/handle/10486/661571

Velázquez, R., García, C., Díaz, J., Rodríguez, J., y Ávila, S. (2014). Estrés y burnout en docentes de educación media superior. Revista Electrónica Medicina, Salud y Sociedad, 4 (2), 1-23. 